\title{
NUMERICAL SOLUTION OF MHD NANOFLUID OVER A STRETCHING SURFACE WITH CHEMICAL REACTION AND VISCOUS DISSIPATION
}

\author{
G. Narender ${ }^{*}$, Santoshi Misra ${ }^{2}$ and K. Govardhan ${ }^{3}$ \\ ${ }^{I}$ Department of H\&S(Mathematics), CVR College of Engineering, Hyderabad, T.S., India \\ ${ }^{2}$ Departmet of Mathematics, St. Ann's College for Women, Hyderabad, T.S., India \\ ${ }^{3}$ Departmetof Mathematics, GITAM University, Hyderabad Campus, T.S., India
}

Received: 28 March 2019 received in revised form17 September 2019

\begin{abstract}
The main objective of this paper is to focus on a numerical study of chemical reaction and viscous dissipation effects on the steady state boundary layer flow of MHD nanofluid past the horizontally stretching sheet with the existence of nanoparticles. A proper similarity transformation is utilized to convert the boundary layer equations into the nonlinear and coupled ordinary differential equations. These ODEs are sorted out numerically by applying the shooting mechanism. Graphical representations are also included to explain the effect of evolving parameters against the abovementioned distributions. Significance of different physical parameters on dimensionless velocity, temperature and concentration are elaborated through graphs and tables. For increasing values of Eckert number, the temperature profile increases whereas the chemical reaction parameter increases, the boundary layer thickness decreases.
\end{abstract}

Keywords: MHD Nanofluid, Chemical reaction parameter; Stretching sheet; Eckert number; Velocity slip Parameter.

DOI: https://doi.org/10.3329/cerb.v21i1.47370

\section{Introduction}

The study of flow and heat transfer generated by means of stretching medium has plenty of significance in numerous industrialized developments, (e.g., in the process of rubber and plastic sheets manufacturing, upgrading the solid materials like crystal, turning fibers etc.). The most widely used coolant liquid among them is water. In above cases, flow and heat transfer investigation is of major importance because final product quality be determined to bulk level based on coefficient of skin friction and heat transfer surface rate. Numerous investigators talked over different traits of stretching flow problem. Some of them are Crane [1], Chaim [2], Liao and Pop [3], Khan and Sanjayanand [4] and T. Fang et al. [5].

Currently, in the fields of engineering and fluid science, heat transfer and boundary layer flow of nanofluid are the thrust areas of research. Many researchers examined the convective boundary layer flow of nanofluid past a stretched sheet. In future, advancement in nano-technology is expected for making unbelievable changes in our lives. A very big number of researchers are working in this area due to its great use in the engineering and its linked areas. In the process of air cleaning, development of microelectronics, safety of nuclear reactors etc., heat and mass transfer of thermophoretic magnetohydrodynamic flow consumes prospective uses. Choi [6] was the first who introduced the idea of "nanofluids" and presented the report on the heat transfer properties of nano-fluids. The thorough exposure on thermophoretic flow was examined by Derjaguin and Yalamov [7]. Heat and mass transfer of MHD thermophoretic stream above plane surface was also studied by Issac and Chamkha [8]. Thermophoresis effect on aerosol particles was investigated by Tsai [9].

In fluid temperature, no doubt, viscous dissipation produces a considerable ascend. This would happen

*Corresponding author email: gnriimc@gmail.com 
because of change in kinetic motion of fluid into thermal energy. Viscous dissipation is unavoidable in case of flow field in high gravitational field. Viscous flow past a nonlinearly stretching sheet was deliberated by Vajravelu [10]. For external natural convention flow over a stretching medium, the effect of viscous dissipation was also studied by Mollendroff and Gebhart [11], whereas the impact of Joule heating and viscous dissipation on the forced convection flow with thermal radiation was presented by Duwairi [12].

The chemical reaction with the diffusion of species for the boundary layer fluid have numerous applications in atmosphere pollution, water, fluids relevant to atmosphere and many other problems of chemical engineering. For boundary layer laminar flow of reactive chemically species with the diffusion which are used by a body over the surface considered by Chamber and Young [13]. For non-Newtonian fluids and their solution for the species of diffusion with chemical reactive in a flow over a stretching sheet with porous medium reported by Akyildiz et al. [14]. Cortell [15] also discuss the two types of viscoelastic fluid over a porous stretching sheet with the chemically reactive species. Hiemenz flow through porous media considered by Chamka and Khaled [16] with the presence of magnetic field. Heat transfer with steady condition considered by Sriramalu et al. [17] for incompressible viscous fluid with porous type species over a stretching surface. Khan et al. [18] discussed MHD viscoelastic fluid, transfer of mass and heat over a permeable stretching surface with stress work and energy dissipation. The fluid on stretching surface close with stagnation-point discussed by Tripathy et al. [19]. Seddeek and Salem [20] observed that the mass and heat transfer distribution on stretching type surface with thermal diffusivity and variable viscosity. M. Ali et al. [21] studied the effects of magnetohydrodynamic on heat transfer and convective mass flow. They concluded that mass flux of particle is as small as the stream velocity and temperature profile are not influenced by thermophysical phenomenon experienced by relatively small number of particles. Some further studies related to magnetohydrodynamic have been discussed in Refs. [22,23,24].

In this article, the findings of M. G. Reddy [25] have been reproduced and extended by considering the viscous dissipation and chemical reaction. The acquired arrangement of partial differential equations is transformed into non-linear and coupled ordinary differential equations by using a similarity transformation. With the help of shooting technique, numerical solution of the system of ordinary differential equations is achieved.

\section{Problem Formulation}

Consider the numerical investigation of MHD boundary layer flow of anin compressible nanofluid. The flow is two-dimensional, steady, laminar, viscous flow of an electrically conducting electrically conducting fluid towards a stretching surface with chemically reactive species undergoing chemical reaction is considered. The schematic diagram of the system under investigation is shown in the Figure 1.

The plate has been stretched with velocity $u_{w}(x)=$ $a x,(a>0)$ along $x$ - direction. In addition, fluid is flowing in the presence of magnetic field.The magnetic field is supposed to be applied along the $y$-direction. The temperature at surface is $T_{f}$, $u_{w}, C_{w}$ represent fluid velocity, nanoparticle concentration at surface respectively.

The following systems of equations are incorporated for mathematical model [25]. shows that in the presence of magnetic field over the surface, the governing equations of conservation of momentum, energy, mass and nanoparticle fraction, under the boundary layer approximation, are as follows:

\section{Continuity equation:}

$\frac{\partial u}{\partial x}+\frac{\partial v}{\partial y}=0$

Momentum equation:

$u \frac{\partial u}{\partial x}+v \frac{\partial u}{\partial y}=-\frac{1}{\rho_{f}} \frac{\partial p}{\partial x}-\frac{\sigma B_{0}^{2}(x)}{\rho_{f}}(u)$

$+v\left(\frac{\partial^{2} u}{\partial x^{2}}+\frac{\partial^{2} u}{\partial y^{2}}\right)$ 


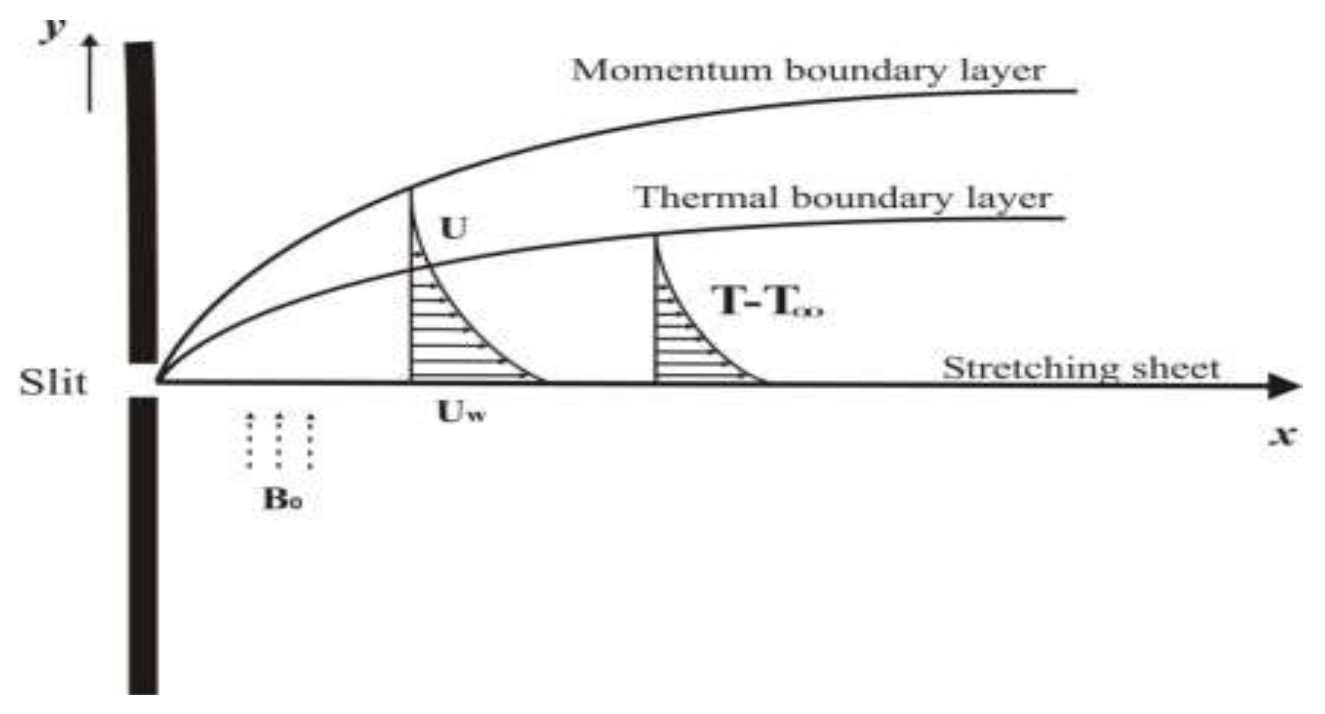

Figure 1: Geometry of the flow under consideration.

$u \frac{\partial v}{\partial x}+v \frac{\partial v}{\partial y}=-\frac{1}{\rho_{f}} \frac{\partial p}{\partial y}-\frac{\sigma B_{0}^{2}(x)}{\rho_{f}}(v)+v\left(\frac{\partial^{2} v}{\partial x^{2}}+\frac{\partial^{2} v}{\partial y^{2}}\right)$

\section{Energy equation:}

$u \frac{\partial T}{\partial x}+v \frac{\partial T}{\partial y}=\frac{\mu}{C_{p}}\left[\left(\frac{\partial u}{\partial x}\right)^{2}+\left(\frac{\partial u}{\partial y}\right)^{2}\right]+\alpha\left[\frac{\partial^{2} T}{\partial x^{2}}+\frac{\partial^{2} T}{\partial y^{2}}\right]+$ $\tau\left\{D_{B}\left(\frac{\partial C}{\partial x} \frac{\partial T}{\partial x}+\frac{\partial C}{\partial y} \frac{\partial T}{\partial y}\right)+\frac{D_{T}}{T_{\infty}}\left[\left(\frac{\partial T}{\partial x}\right)^{2}+\left(\frac{\partial T}{\partial y}\right)^{2}\right]\right\}-\frac{\alpha}{\rho_{f}}\left(\frac{\partial q_{r}}{\partial y}\right)$

\section{Concentration equation:}

$u \frac{\partial C}{\partial x}+v \frac{\partial C}{\partial y}=D_{B}\left(\frac{\partial^{2} C}{\partial x^{2}}+\frac{\partial^{2} C}{\partial y^{2}}\right)+\frac{D_{T}}{T_{\infty}}\left(\frac{\partial^{2} C}{\partial x^{2}}+\frac{\partial^{2} C}{\partial y^{2}}\right)-$ $k_{0}\left(C-C_{\infty}\right)$

where $u$ and $v$ are the components of velocity respectively in the $x$ and $y$ directions, $p$ is the fluid pressure, $\rho_{f}$ is the density of base fluid, $\rho_{p}$ is the density of the particles, $v$ is the kinematic viscosity of the base fluid, $\sigma$ is electrical conductivity, $\rho$ is density, $T$ is temperature, $\alpha$ is thermal diffusivity, $\tau=\frac{(\rho c)_{p}}{(\rho c)_{f}}$ is the ratio of nanoparticle heat capacity and the base fluid heat capacity, $D_{B}$ is Brownian diffusion coefficient, $D_{T}$ is thermophoretic diffusion coefficient, $\mu$ is dynamic viscosity, $c$ is specific heat at constant pressure. The boundary conditions for Eqs. $(1-5)$ are

$$
\left.\begin{array}{c}
u=u_{w}+L \frac{\partial u}{\partial y}, v=v_{w}, \\
-k \frac{\partial T}{\partial y}=h\left(T_{f}-T\right), \\
C=C_{w} \text { at } y=0 \\
u=0, v=0, T \rightarrow T_{\infty}, C \rightarrow C_{\infty} \text { as } y \rightarrow \infty
\end{array}\right\}
$$

The radiative heat flux $q_{r}$, by using the Rosseland approximation for radiation, can be written as

$q_{r}=\frac{-4 \sigma^{*}}{3 k^{*}} \frac{\partial T^{4}}{\partial y}$

where $\sigma^{*}$ and $k^{*}$ stand for the Stefan-Boltzmann constant and coefficient of mean absorption. Expansion of $T^{4}$ about $T_{\infty}$ by making use of Taylor's series is:

$T^{4}=T_{\infty}^{4}+\frac{4 T_{\infty}^{3}}{1 !}\left(T-T_{\infty}\right)^{1}+\frac{12 T_{\infty}^{2}}{2 !}\left(T-T_{\infty}\right)^{2}+$ $\frac{24 T_{\infty}}{3 !}\left(T-T_{\infty}\right)^{3}+\frac{24}{4 !}\left(T-T_{\infty}\right)^{4}$

Disregarding the higher order terms, $T^{4}=T_{\infty}^{4}+$ $4 T_{\infty}^{3}\left(T-T_{\infty}\right)$

$\Rightarrow \frac{\partial T^{4}}{\partial y}=4 T^{3} \frac{\partial T}{\partial y}$

Using (9) in (7) and the differentiate w.r.t.y, we get $\frac{\partial q_{r}}{\partial y}=-\frac{16 \sigma^{*} T_{\infty}^{3}}{3 \kappa^{*}} \frac{\partial^{2} T}{\partial y^{2}}$

We use similarity transformation to solve Eqs. (1 5)

$$
\begin{aligned}
& \psi=\sqrt{a v} \times f(\eta), \theta(\eta)=\frac{T-T_{m}}{T_{\infty}-T_{m}}, \\
& \beta(\eta)=\frac{c-C_{\infty}}{C_{w}-C_{\infty}}, \quad \eta=y \sqrt{\frac{a}{v}},
\end{aligned}
$$

the velocity component of stream function which is defined as 
$u=\frac{\partial \psi}{\partial y}, v=-\frac{\partial \psi}{\partial x}$

So, we have

$u=a x f^{\prime}(\eta), \quad u=-\sqrt{a v} f(\eta)$

where prime shows differentiation with respect to $\eta$.

The equation continuity (1) is satisfied identically. The governing Eqs. (2) - (5) are reduced into the following nonlinear ODEs.

$f^{\prime \prime \prime}+f f^{\prime \prime}-\left(f^{\prime}\right)^{2}-M f^{\prime}=0$

$\left(1+\frac{4}{3} R\right) \theta^{\prime \prime}+\operatorname{Pr} \bar{p} f \theta^{\prime}+N b \theta^{\prime} \beta^{\prime}+N t\left(\theta^{\prime}\right)^{2}+$

$\left.E c\left(f^{\prime \prime}\right)^{2}\right]=0$

$\beta^{\prime \prime}+\operatorname{Lef} \beta^{\prime}+\frac{N t}{N b} \theta^{\prime \prime}-\operatorname{Le\gamma } \beta=0$

with the boundary conditions

$$
\left.\begin{array}{c}
f(0)=S, f^{\prime}(0)=1+\mathrm{A} f^{\prime \prime}(0), \\
\theta^{\prime}(0)=-B i(1-\theta(0)),=0, \beta(0)=1 \\
\text { at } \eta=0, \\
f^{\prime}(\infty) \rightarrow 0, \theta(\infty) \rightarrow 0, \beta(\infty) \rightarrow 0 \\
\text { as } \eta \rightarrow \infty
\end{array}\right\}
$$

The dimensionless constants $M, S, \mathrm{~A}, R, \mathrm{Pr}, N b$, $N t, E c, L e, \gamma, B i$ represent the magnetic parameter, a suction parameter,the velocity slip parameter, the radiation parameter, the Prandtl number, the Brownian motion parameter, the thermophoresis parameter, the Eckert number, the Lewis number, the chemical rate parameter, the Biot number respectively, which are defined as,

$M=\frac{\sigma B_{0}^{2}}{\rho a}, S=-\frac{v_{w}}{\sqrt{a v}}, A=L\left(\sqrt{\frac{a}{v}}\right)$,

$R=\frac{-4 T_{\infty}^{3} \sigma^{*}}{3 k^{*} k}, \operatorname{Pr}=\frac{v}{\alpha}$,

$N b=\frac{\tau D_{B}\left(C_{w}-C_{\infty}\right)}{v}$,

$N t=\frac{\tau D_{T}\left(T_{f}-T_{\infty}\right)}{v T_{\infty}}, E c=\frac{u_{w}^{2}}{\rho_{f}\left(T_{f}-T_{\infty}\right)}$

$L e=\frac{v}{D_{B}}, \gamma=\frac{k_{0} U\left(C_{w}-C_{\infty}\right)}{v}, B i=\frac{h(v / a)^{1 / 2}}{k}$.

Nusselt number $N u_{x}$, skin friction $\operatorname{coefficient} C_{f}$, and Sherwood number $S h_{x}$, are expressed as:

$N u_{x}=\frac{x q_{w}}{k\left(\left(T_{w}-T_{\infty}\right)\right.}, C_{f}=\frac{\tau_{w}}{\rho U_{w}^{2}}$, and
$S h_{x}=\frac{x q_{m}}{D_{B}\left(\left(C_{w}-C_{\infty}\right)\right.}$

where, $\tau_{w}$ is the shear stress, $q_{m}$ is the wall mass flux from the surface, and $q_{w}$ is the heat flux at the wall surface, given by:

$q_{w}=-k\left(\frac{\partial T}{\partial y}\right)_{y=0}, q_{m}=-D_{B}\left(\frac{\partial C}{\partial y}\right)_{y=0}$

Using the dimensionless variables, we get

$\frac{N u_{x}}{\sqrt{R_{x}}}=-1+R \theta^{\prime}(0), \frac{S h_{x}}{\sqrt{R_{x}}}-\beta^{\prime}(0)$,

$C_{f} \sqrt{R_{x}}=-f^{\prime \prime}(0)$

where $R e_{x}$ denotes the Reynolds number and is expressed as:

$R e_{x}=\frac{x U_{w}(x)}{v}$.

\section{Numerical Treatment}

In this section, the scheme for the numerical solution of the system of three coupled ODEs (14) - (16) with BCs (17) will be discussed. Because of its efficiency, the shooting technique has been performed to apply. Firstly, it is noticed that heurist infinity for the independent variable chosen as $\eta_{\max }$.

The comment on the choice on the $\eta_{\max }$, for solving is presented at the end of the section.

Equation (14) is solved with $f^{\prime \prime}(0)=r$, assumed number using the initial conditions

$f(0)=S, f^{\prime}(0)=1+\lambda \times r, f^{\prime \prime}(0)=r$

$r$ is iteratively found using Newton's method using $F^{\prime}\left(\eta_{\max }\right)=\frac{\partial f^{\prime}(\max )}{\partial \alpha}$ which is obtain by solving,

$F^{\prime \prime \prime}=2 f^{\prime} F^{\prime}-f F^{\prime \prime}-f^{\prime \prime} F^{\prime}+M f^{\prime}$

with $F(0)=0, F^{\prime}(0)=\lambda$ and $F^{\prime \prime}(0)=1$

After finding $f(\eta)$ we solved the equations (15) and (16) with the initial conditions.

$$
\left(\begin{array}{l}
\mathrm{y}_{1}^{\prime} \\
\mathrm{y}_{2}^{\prime} \\
\mathrm{y}_{3}^{\prime} \\
\mathrm{y}_{4}^{\prime}
\end{array}\right)=\left(\begin{array}{c}
\mathrm{y}_{2} \\
\frac{-\operatorname{Pr}\left(f \mathrm{y}_{2}+\mathrm{Nb} \mathrm{y}_{2} \mathrm{y}_{4}+\mathrm{Nty} \mathrm{y}_{2}^{2}+\mathrm{Ec} f^{\prime \prime 2}\right)}{\left(1+\frac{4 \mathrm{R}}{3}\right)} \\
\mathrm{y}_{4} \\
-\mathrm{Le} f \mathrm{y}_{4}-\frac{\mathrm{Nb}}{\mathrm{Nt}} \mathrm{y}_{2}^{1}+\mathrm{Le} \gamma \mathrm{y}_{3}
\end{array}\right)
$$


Associated boundary conditions in Eq. (17) can be written as

$$
\left(\begin{array}{l}
\mathrm{y}_{1}(0) \\
\mathrm{y}_{2}(0) \\
\mathrm{y}_{3}(0) \\
\mathrm{y}_{4}(0)
\end{array}\right)=\left(\begin{array}{c}
\mathrm{p}_{1} \\
B i\left(\mathrm{p}_{1}-1\right) \\
1 \\
\mathrm{p}_{2}
\end{array}\right)
$$

Here $p_{1}=\theta(0)$ and $p_{2}(0)=\beta^{\prime}(0)$.

$p_{1}, p_{2}$ are to be found satisfying end conditions $y_{1} \rightarrow 0, y_{3} \rightarrow 0$ as $\eta \rightarrow \infty$. Adams Moultan fourth order method (with the corresponding predictor) is used to solve the initial value problem. Assumed values of $p_{1}$ and $p_{2}$ are corrected using Newton method. Derivatives of $\theta\left(\infty, p_{1}, p_{2}\right)$ and

$\phi\left(\infty, p_{1}, p_{2}\right) \quad$ with respect to any parameter $p\left(p_{1}\right.$ or $\left.p_{2}\right)$ are found by solving the equation which are obtained by differentiating system (21). $Y_{i}=\frac{\partial y_{i}}{\partial p}$ for all $i=1,2,3,4$. These equations are

$Y_{1}^{\prime}=Y(2)$,

$Y_{2}^{\prime}=\frac{-P r}{\left(1+\frac{4 R a}{3}\right)}[F F(I) Y(2)+N b(y(2) Y(4)-$

Y2y4)+2Nty2Y2,

$Y_{3}^{\prime}=Y(4)$,

$Y_{4}^{\prime}=-\operatorname{LeFF}(I) y(4)-\frac{N t}{N b} Y_{2}^{\prime}+\operatorname{Le\gamma y}(3)$

This system is solved with three different sets of initial conditions $y_{i}(0)=0$ for all $i=1,2,3,4$.

Newton's method is

$$
\left(\begin{array}{l}
p_{1} \\
p_{2}
\end{array}\right)^{\text {New }}=\left(\begin{array}{l}
p_{1} \\
p_{2}
\end{array}\right)^{\text {old }}-\left[\begin{array}{ll}
\frac{\partial y_{1}}{\partial p_{1}} & \frac{\partial y_{1}}{\partial p_{2}} \\
\frac{\partial y_{3}}{\partial p_{1}} & \frac{\partial y_{3}}{\partial p_{2}}
\end{array}\right]_{\eta=\infty}^{-1}\left(\begin{array}{l}
y_{1} \\
y_{3}
\end{array}\right)
$$

It may be noticed that the choice of initial guess of $p_{1}, p_{2}$ is very crucial. Once we obtain solution for a set of physical parameters, a single parameter changed slightly to achieve convergence of Newton's method. The choice of $\eta_{\max }=6$ was more than enough for end condition. The convergence criteria are chosen to be successive value agree up to 3 significant digits.

\subsection{Code Validation}

For the validity of our results, the skin friction factor, the local Nusselt number and Sherwood number have been compared with those already published in literature as shown in Tables 3.1 and 3.2. From tables the results achieved by the present code are found convincingly very closed to the published results [25].

Table 3.1. Comparison of results of $-f^{\prime \prime}(0)$ for various values of $M, S$ and $A$

\begin{tabular}{ccccc}
\hline \multirow{2}{M}{} & \multirow{2}{*}{$\boldsymbol{S}$} & $\boldsymbol{A}$ & \multicolumn{2}{c}{$-\boldsymbol{f}^{\prime \prime}(\mathbf{0})$} \\
\cline { 4 - 5 } & & & M. G. Reddy [25] & Present value \\
\hline $\mathbf{0 . 5}$ & 0.2 & 0.1 & 1.13992 & 1.136756 \\
\hline $\mathbf{1 . 0}$ & 0.2 & 0.1 & 1.28430 & 1.282955 \\
\hline $\mathbf{0 . 5}$ & 0.5 & 0.1 & 1.26830 & 1.265184 \\
\hline $\mathbf{0 . 5}$ & 0.2 & 0.5 & 0.800331 & 0.8066351 \\
\hline
\end{tabular}

Table 3.2. Comparison of results of $-\theta^{\prime}(0)$ and $-\beta^{\prime}(0)$

\begin{tabular}{cccccc}
\hline & & \multicolumn{2}{c}{$-\boldsymbol{\theta}^{\prime}(\mathbf{0})$} & \multicolumn{2}{c}{$-\boldsymbol{\beta}^{\prime}(\mathbf{0})$} \\
\cline { 3 - 6 } Nt & $\boldsymbol{N} \boldsymbol{b}$ & $\begin{array}{c}\text { M. G. } \\
\text { Reddy } \\
\text { [25] }\end{array}$ & $\begin{array}{c}\text { Present } \\
\text { value }\end{array}$ & $\begin{array}{c}\text { M. G. } \\
\text { Reddy } \\
{[25]}\end{array}$ & $\begin{array}{c}\text { Present } \\
\text { value }\end{array}$ \\
\hline $\mathbf{0 . 1}$ & 0.1 & $\begin{array}{c}0.08406 \\
76\end{array}$ & $\begin{array}{c}0.0822515 \\
300\end{array}$ & 2.96584 & 2.9588820 \\
\hline $\mathbf{0 . 5}$ & 0.1 & $\begin{array}{c}0.08139 \\
44\end{array}$ & $\begin{array}{c}0.0792839 \\
800\end{array}$ & 3.00253 & 3.0008590 \\
\hline $\mathbf{0 . 1}$ & 0.5 & $\begin{array}{c}0.08384 \\
97\end{array}$ & $\begin{array}{c}0.0819882 \\
600\end{array}$ & 2.79651 & 2.7606710 \\
\hline $\mathbf{0 . 5}$ & 0.5 & $\begin{array}{c}0.08110 \\
44\end{array}$ & $\begin{array}{c}0.0789362 \\
500\end{array}$ & 2.97637 & 2.9675340 \\
\hline
\end{tabular}

\section{Graphical Results}

The objective is to inspect governing parameters on the velocity, temperature and concentration distribution in this section. In every one of these estimations, we have considered $\operatorname{Pr}=2, M=2, R=$ $2, N b=N t=S=A=E c=\gamma=0.5, B i=0.1 \quad$ and $L e=5$.

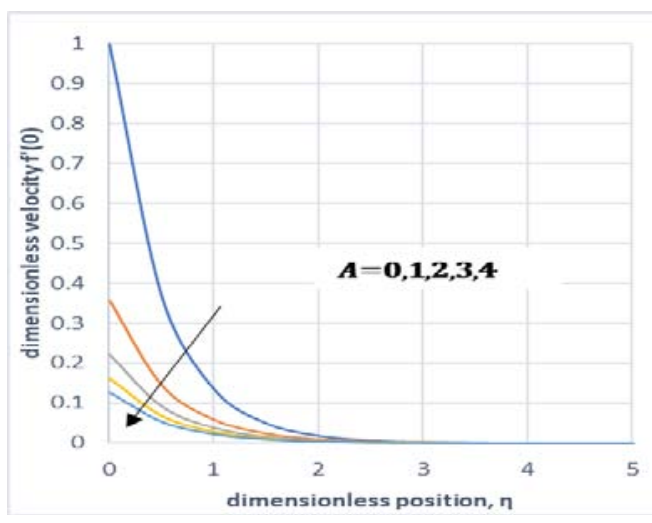

Figure 2. Dimensionless Velocity vs $A$ 


\subsection{Impact of slip parameter $(A)$.}

The effect of slip parameter $A$ on the dimensionless velocity profile $f^{\prime}(\eta)$ is presented in Figure2. Increasing the values of the slip parameter $A$ reduces the velocity field and boundary thickness as depicted in Figure 2.

\subsection{Impact of Magnetic parameter $(M)$.}

Figure 3 shows the behavior of $M$ on velocity profile it is noticed the effects of magnetic field are to reduce the velocity profile. Because of the application of transverse magnetic field in an electrically conducting fluid, a resistive force like a drag force is produced, which is Lorentz force.

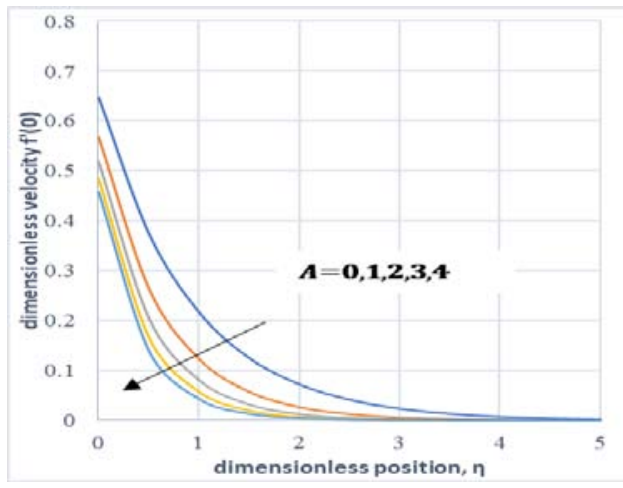

Figure 3. Dimensionless Velocity vs $M$

The presence of Lorentz force retards the force on the velocity field. Figure. 4 and Figure. 5 shows the variations in energy profile $\theta(\eta)$ and dimensionless concentration profile $\beta(\eta)$ for different estimations of Magnetic Parameter $M$. It is analyzed that the temperature profile $\theta(\eta)$, concentration profile $\beta(\eta)$ and thermal boundary layer thickens are increasing functions of Magnetic Parameter.

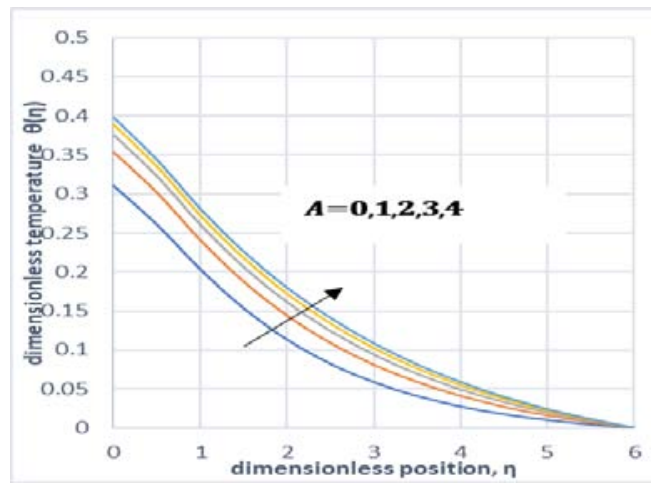

Figure 4. Dimensionless Temperature vs $M$
The reason beyond this electrically conducting fluid produces a resistive force known as Lorentz force, which opposes the flow and tends to make the fluid motion slowdown in the boundary layer and therefore reduces the profile of temperature $\theta(\eta)$ increases with the increase in magnetic parameter.

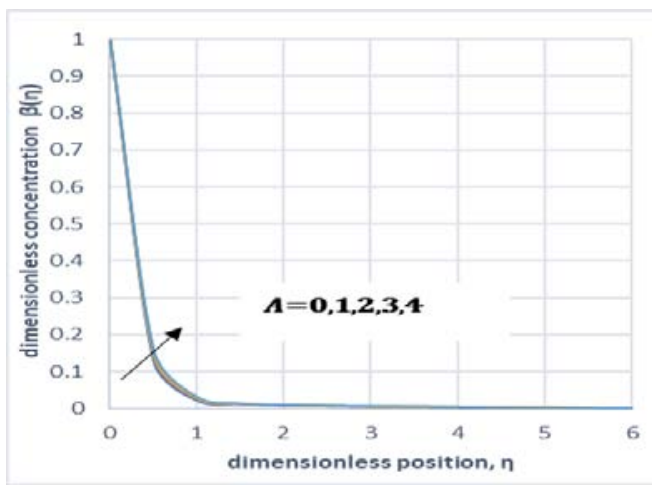

Figure 5. Dimensionless Concentration vs $M$

\subsection{Impact of suction parameter $(S)$.}

The impact of the section parameter $S$ on the dimensionless velocity profile $f^{\prime}(\eta)$ ispresented in Figure 6. Velocity profile diminishes and accompanied with boundary layer width increases for gradually growing values of the suction parameterS.Figure 7 displays the variation of temperature with suction parameter. As the values of suction parameter $S$ increase, the temperature graph is decreasing. Moreover, the thermal boundary layer thickness and surface temperature is also decreasing. Figure 8 shows that by increasing suction parameter $S$, concentration profile decreases. By an increasing in the suction, concentration profile as wellas boundary layer thickness decrease.

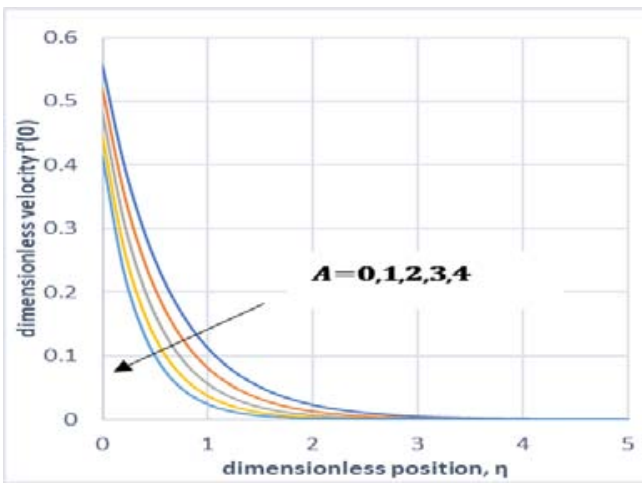

Figure 6. Dimensionless Temperature vs $S$ 


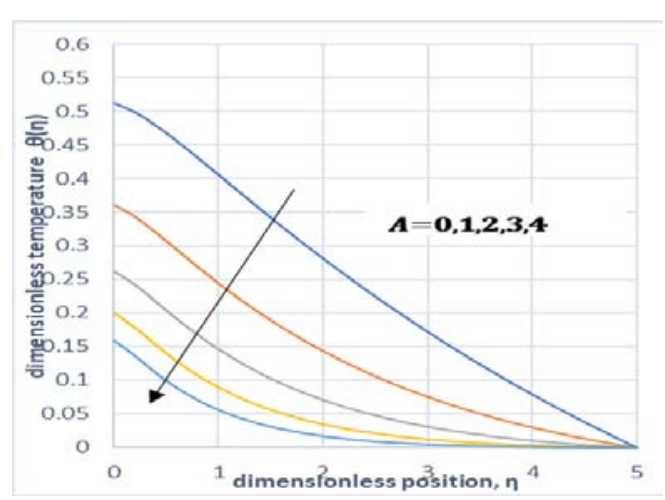

Figure 7. Dimensionless Temperature vs $S$

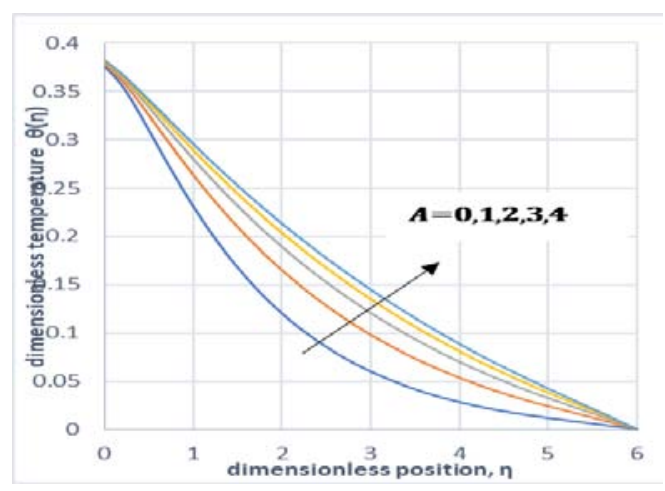

Figure 8. Dimensionless Temperature vs $S$

\subsection{Impact of Prandtl number $(P r)$.}

Figure 9 presents that an elevation in Prandtl number $\operatorname{Pr}$ shows a reduction in the temperature profile $\theta(\eta)$. Obviously, greater Prandtl number $\operatorname{Pr}$ has weaker thermal diffusivity due to which low range temperature. This indicates resection in energy exchange ability and finally it causes a reduction in thermal boundary surface.

\subsection{Impact of Radiation parameter $(R)$.}

The influence of radiation parameter on profile of temperature distribution is displayed in Figure 10. From the figure it is observed that by increasing the radiation parameter, temperature profile decreases significantly. It is because the increasing values of radiation parameter lead to decrease the thickness of the boundary layer and enhance the heat transfer rate with chemical effect on the melting surface.

\subsection{Impact of Convective Heating $(B i)$.}

Figures 11 is presented to visualize the effect of the $B i$ on temperature distribution. This figure defines that temperature profile enhances as the Biot number is increased gradually. Physically, the Biot number defines the ratio between resistance rate of heat transfer inside the body to the resistance at the body surface. The reason behind is that convective heat exchange at the surface will raise the boundary layer thickness therefore the nanofluid with convective boundary condition is more effective model as compared to the constant surface temperature state.

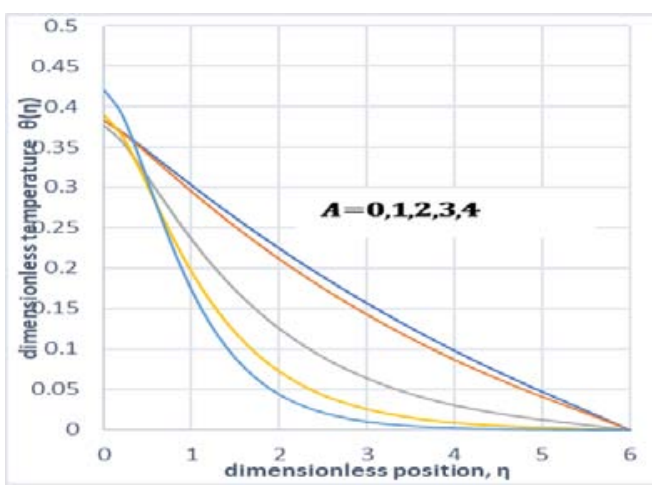

Figure 9. Dimensionless Temperature vs $\mathrm{Pr}$

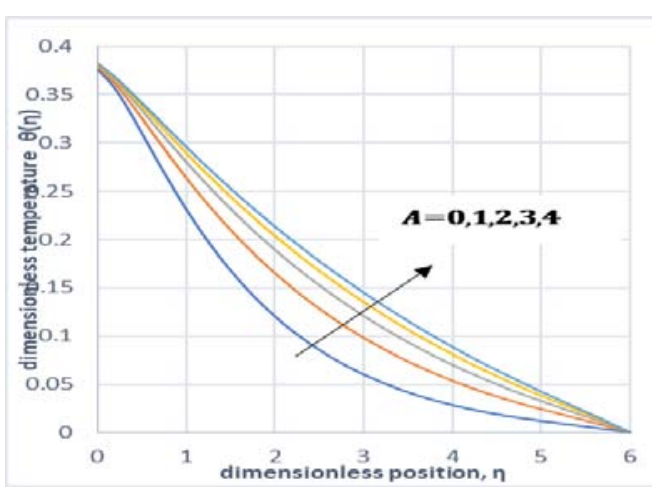

Figure 10. Dimensionless Temperature vs $R$

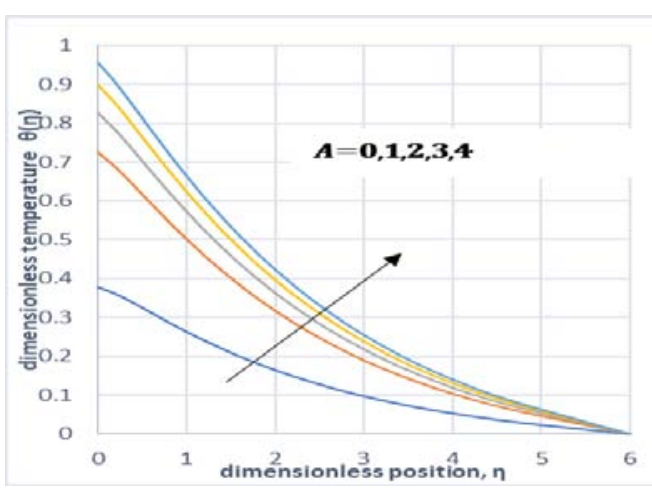

Figure 11. Dimensionless Temperature vs Bi 


\subsection{Impact of Brownian motion and the thermophoresis on unitless energy profile}

Figure 12 shows the impact of Brownian motion and the thermophoresis parameters on the temperature distributions $\theta(\eta)$ in the thermal boundary layer. Thermophoresis is a component that drives small materials away from hot layer to the cooler end. It is noticed that as thermophoresis parameter increases the thermal boundary layer thickness increases and the temperature gradient at the surface decrease (in absolute value) as both $\mathrm{Nb}$ and $N t$ increase.

\subsection{Impact of Lewis number (Le)}

Lewis number $L e$ is decreasing function of $\beta(\eta)$. Increase Lewis number may decrease Brownian diffusion coefficient because Lewis number is the ratio of momentum diffusivity to Brownian diffusion coefficient, shown in Figure 13.

\subsection{Impact of Eckert number $(E c)$.}

Figure 14 displays the influence of $E c$ on the energy profile. Energy profile increases when the Eckert number is increased. Due to friction, the heat energy is kept in owing to accelerating values of $E c$, which results in the enhancement of the temperature profile.

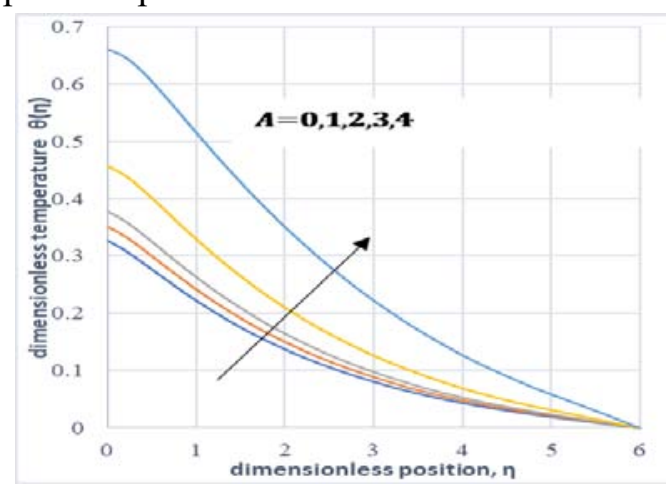

Figure12. Dimensionless Temperaturevs $N t$ and $\mathrm{Nb}$

\subsection{Impact of chemical reaction parameter $(\gamma)$}

Figure 15explains the influence of the chemical reaction parameter on the profile of concentration. It is noted that increasing values of chemical reaction parameter concentration as well as the thickness of concentration decrease. It is because the chemical reaction in this system results in chemical dissipation and therefore results in decrease in the profile of concentration. The most significant influence is that chemical reaction tends to increase the overshoot in the concentration profiles and their associated boundary layer.

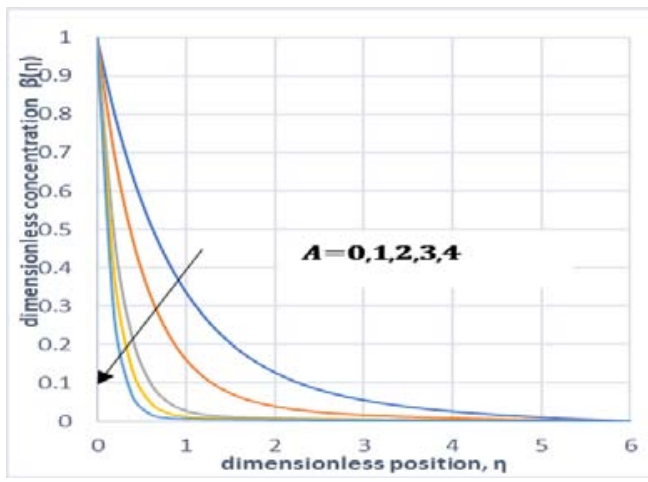

Figure 13. Dimensionless Concentration vs Le

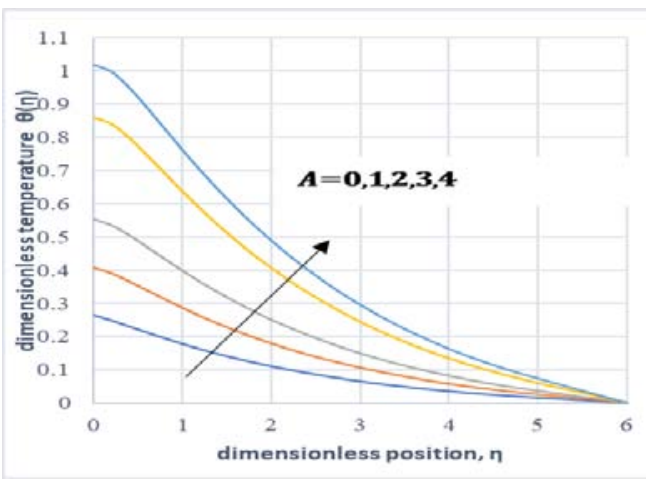

Figure14. Dimensionless Temperature vs Ec

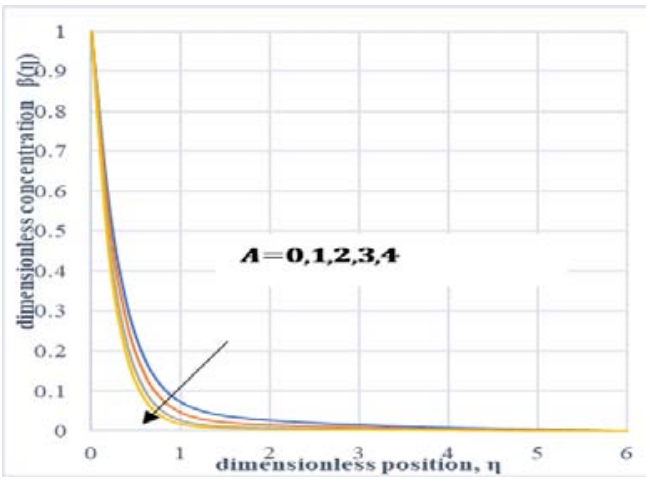

Figure 15. Dimensionless Concentration vs $\gamma$

\section{Conclusion}

From the above discussion, we can make the following conclusions.

Higher values of $M$ yield an increment in the energy profile and Concentration profile whereas an opposite effect has noticed for the velocity profile. Because of the presence of Lorentz force 
retards the force on the velocity field and his force has the tendency to slow down the fluid motion.

By increasing in the suction parameter $S$, velocity profile increases whereas temperature profile decreases with an increase in suction while due to increasing blowing, it increases. The thickness of concentration boundary layers reduces due to increasing in the suction parameter. Increase of Prandtl number Prand Radiation parameter $R$ causes decrease in temperature profile. Due to increase in thermal Radiation parameter more heat to fluid produces that enhance the energy filed and momentum boundary layer. Temperature increase by enlarging thermophoresis parameter $N t$ and Brownian motion parameter $N b$. Physically, heated particles come away from high temperature as compared to low-temperature so the temperature of fluid increases. It is reported that for enhancing values of the Biot number temperature profile enhanced. Physically, the Biot number is basically the ratio between resistances of the heat transfer in the body to the resistance at body surface. This is because convective heat exchange along the surface will enhance the momentum boundary layer. For larger values of Lewis number $L e$ and chemical reaction parameter, concentration field $\beta(\eta)$ shows decreasing behavior. Temperature profile will increase due to increases in the Eckert number.

\section{Acknowledgment}

The suggestions of reviewer regarding an earlier version are appreciated.

\section{Nomenclature}

$\begin{array}{ll}B & \text { Magnetic field strength }\left(\mathrm{Wbm}^{-2}\right) \\ a & \text { Constant }\left(\mathrm{s}^{-1}\right) \\ D_{B} & \text { Brownian diffusion coefficient } \\ D_{T} & \text { Thermophoretic diffusion coefficient } \\ \kappa & \text { Thermal conductivity }\left(\mathrm{Wm}^{-1} \mathrm{~K}^{-1}\right) \\ \sigma^{*} & \text { Stefan-Boltzmann constant }\left(\mathrm{kgm}^{-2} \mathrm{~K}^{-4}\right) \\ k^{*} & \text { Mean absorption } \\ E c & \text { Eckert number } \\ L e & \text { Lewis number } \\ M & \text { Magnetic parameter } \\ \mathrm{Nb} & \text { Brownian motion parameter } \\ \mathrm{Nt} & \text { Thermophoresis parameter } \\ \mathrm{Nu} & \text { Nusselt number }\end{array}$

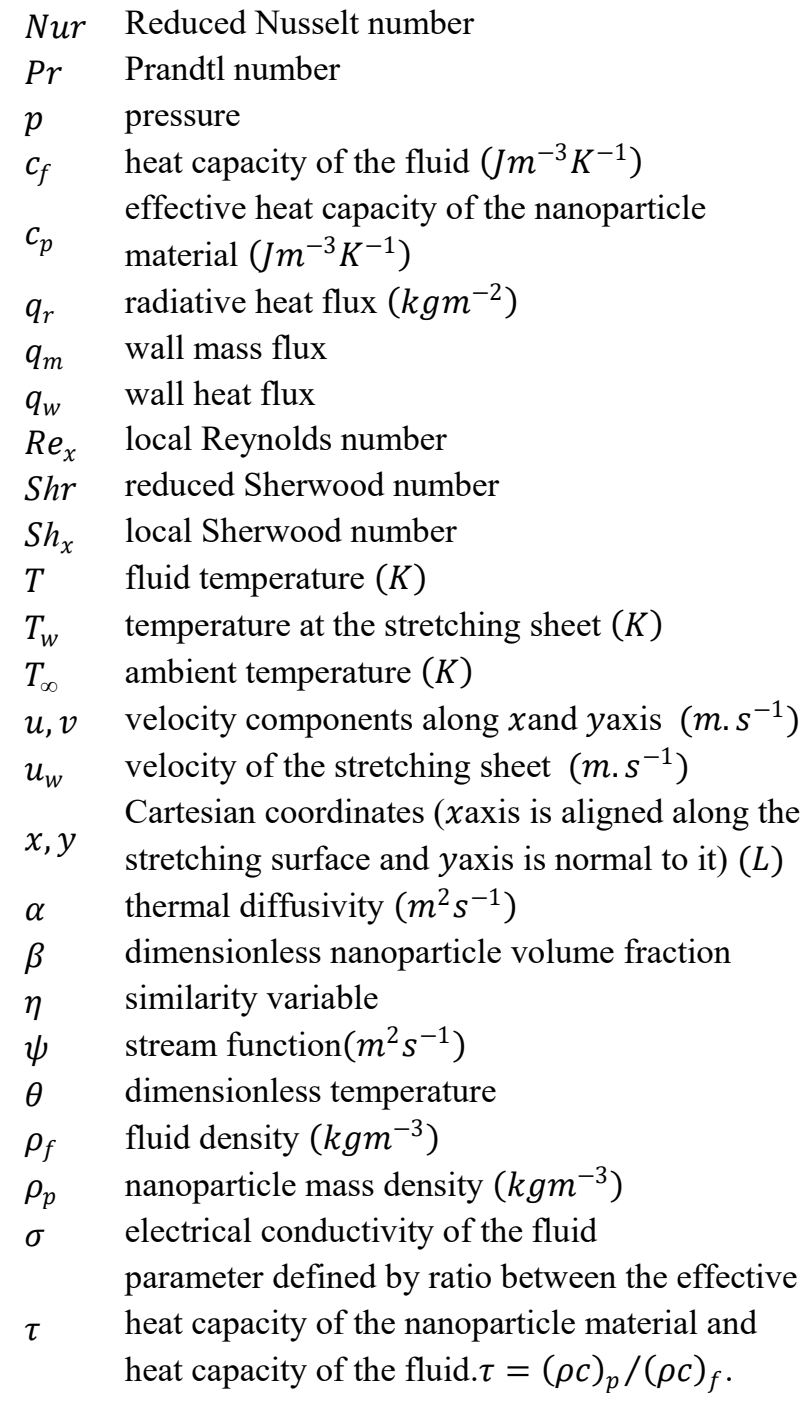

\section{References}

[1] L. J. Crane. Flow past a stretching plate. Zeitschrift für Angewandte Mathematik und Physik ZAMP, 21(4):645-647, 1970.

[2] T. C. Chaim. Hydromagnetic flow over a surface stretching with a power-law velocity. Int. J. Engineering Science, 33(3):429-435, 1995.

[3] S. J. Liao and I. Pop. Explicit analytic solution for similarity boundary layer equations. Int. J. Heat and Mass Transfer, 47(1):75-85, 2004.

[4] S. K. Khan and E. Sanjayanand. Viscoelastic boundary layer flow and heat transfer over an exponential stretching sheet. Int. J. Heat and Mass Transfer, 48(8):1534-1542, 2005.

[5] T. Fang, F. L. Chiafon, and J. Zhang. The boundary layers of an unsteady incompressible stagnation-point flow with mass transfer. Int. J. Non-Linear Mechanics, 46(7):942-948, 2011. 
[6] S. Choi. Enhancing thermal conductivity of fluids with nanoparticles in developments and applications of non-Newtonian flows, Am. Soc. Mech. Eng., 99-105 1995.

[7] B. V. Derjaguin and Y. Yalamov. Theory of thermophoresis of large aerosol particles. J. Colloid science, 20(6):555-570, 1965.

[8] J. Chamkha and C. Issac. Effects of heat generation/absorption and thermophoresis on hydromagnetic flow with heat and mass transfer over a at surface. Int. J. Numerical Methods for Heat \& Fluid Flow, 10(4):432-449, 2000.

[9] R. Tsai. A simple approach for evaluating the effect of wall suction and thermophoresis on aerosol particle deposition from a laminar flow over a at plate. Int. C. Heat and Mass Transfer, 26(2):249-257, 1999.

[10] K. Vajravelu. Viscous flow over a nonlinearly stretching sheet. Applied mathematics and computation, 124(3):281-288, 2001.

[11] B. Gebhart and J. Mollendorf. Viscous dissipation in external natural convection flows. J. Fluid mechanics, 38(01):97-107, 1969.

[12] H. M. Duwairi. Viscous and Joule heating effects on forced convection flow from radiate isothermal porous surfaces. Int. J. Numerical Methods for Heat \& Fluid Flow, 15(5):429-440, 2005.

[13] P. L. Chamber and J. D. Young. On diffusion of a chemically reactive species in a laminar boundary layer flow. Phys. Fluids, 1:48-54, 1958.

[14] F. T. Akyildiz, H. Bellout, and K. Vajravelu. Diffusion of a chemically reactive species in a porous medium over a stretching sheet. J. Math. Anal. Appl, 320:322-339, 2006.

[15] R. Cortell. Flow and heat transfer of a fluid through a porous medium over a stretching surface with internal heat generation/absorption and suction/blowing. Fluid Dyn. Res, 37:231-245, 2005.

[16] A. J. Chamka and A.R.A.Khaled. Similarity solution for hydromagnetic mixed convection and mass transfer heimntz flow through porous media. In. J. Numer. Methods Heat Fluid Flow, 10:94115, 2000 .

[17] A. Sriramalu, N. Kishan, and A.J. Anand. Steady flow and heat transfer of a viscous incompressible fluid flow through porous medium over a stretching sheet. J. Energy, Heat Mass Transfer, 23:483-495,2001.

[18] S.K. Khan, A.M. Subhas, and R.M. Sonth. Viscoelastic MHD flow, heat and mass transfer over a porous stretching sheet with dissipation energy and stress work. Heat Mass Transfer, 40:47-57, 2003.

[19] R. S. Tripathy, G.C. Dash, S.R. Mishra, and S. Baag. Chemical reaction effect onMHDfreeconvectivesurfaceoveramovingvertical planethroughporousmedium. Alexandria Engineering Journal, 54(3):673-679, 2015.

[20] M.A. Seddeek and A.M. Salem. Laminar mixed convection adjacent to vertical continuously stretching sheet and variable viscosity and variable thermal diffusivity. Heat Mass Transf., 41:10481055, 2005.

[21] M. Ali, M. A. Alim, R. Nasrin, M. S. Alam. Numerical analysis of heat and mass transfer along a stretching wedge surface. Journal of Naval Architecture and Marine Engineering, 1813-8235, December 2017.

[22] M. Ali, M. A. Alim, R. Nasrin, M. S. Alam, M. J. Haque Munshi. Similarity Solution of Unsteady MHD Boundary Layer Flow and Heat Transfer past a Moving Wedge in a Nanofluid using the Buongiorno Model, Procedia Engineering, 194, 407 - 413, 2017.

[23] M. Ali, M. A. Alim, R. Nasrin, and M. S. Alam, Study the effect of chemical reaction and variable viscosity on free convection MHD radiating flow over an inclined plate bounded by porous medium, AIP Conference Proceedings, 1754, 040009, 2016.

[24] M. S. Alam, M. Ali, M. A. Alim, and M. J. Haque Munshi. Unsteady boundary layer nanofluid flow and heat transfer along a porous stretching surface with magnetic field, AIP Conference Proceedings, 1851, 020023, 2017.

[25] M. G. Reddy. Influence of Magneto hydrodynamic and Thermal Radiation Boundary Layer Flow of a Nanofluid Past a Stretching Sheet. J. Sci. Res. 6 (2): 257-272, 2014. 